\title{
Assessment of Effectiveness of Science and Technology Projects: A Case Study of National Science and Technology Programme for Sustainable Development of North West Vietnam
}

\author{
Mai Trong Nhuan ${ }^{1, *}$, Truong Xuan $\mathrm{Cu}^{2}$, Nguyen Thi Hoang $\mathrm{Ha}^{1}$, Tran Dang Quy ${ }^{1}$, \\ Pham Thuy Linh ${ }^{1}$, Nguyen Tai Tue ${ }^{1}$, Luu Viet Dung ${ }^{1}$ \\ ${ }^{I}$ VNU University of Science, Vietnam National University, Hanoi, 334 Nguyen Trai, Hanoi, Vietnam \\ ${ }^{2}$ Party Committee of the Centrally-run Administrative Sector, 105B, Quan Thanh, Hanoi, Vietnam
}

Received 16 September 2019

Revised 11 December 2019; Accepted 22 December 2019

\begin{abstract}
The present research was conducted to propose the index for assessing the effectiveness of science and technology projects in Vietnam. The effectiveness of projects was measured through 20 variables of 03 indicators: (1) The effectiveness of science and technology values including 12 variables; 2 ) The effectiveness of human resources including 04 variables; 3 ) and the effectiveness of education and training including 04 variables. These variables were evaluated by the $0-1$ scale, with the zero-value indicated ineffectiveness of projects and vice versa. These variables were measured by the number of results, products from selected projects, and compared with the actual value in the project contract. Total 8 projects (including natural sciences, social sciences and technology projects) from National Science and Technology Program for Sustainable Development of North West Vietnam (NSTP-SDNW) were selected from 58 projects of NSTP-SDNW for testing the present index. Research results showed that the number of results and products of all projects have been met or exceeded requirements in project contracts. The assessment value of social projects (code 06X, 07X, 17X) ranged from 0.55 to 0.75 , whereas the assessment value of natural sciences and technology projects ranged from 0.55 to 0.72 . Research results showed that all selected projects are measured as high effective level, with the highest effectiveness was observed in No. 06X and No. $02 \mathrm{C}$ projects.
\end{abstract}

Keywords: Assessment, Effectiveness, Project of Science and Technology, Product of Science and Technology, Indicators.

\footnotetext{
* Corresponding author.

E-mail address: nhuanmt@vnu.edu.vn

https://doi.org/10.25073/2588-1094/vnuees.4457
} 


\title{
Đánh giá hiệu quả của các đề tài, dự án, chương trình khoa học và công nghệ: Trường hợp Chương trình Khoa học và Công nghệ phục vụ phát triển bền vững vùng Tây Bắc
}

\author{
Mai Trọng Nhuận ${ }^{1 *}$, Trương Xuân Cừㄹㅅㅜ Nguyễn Thị Hoàng Hà̀ ${ }^{1}$, Trần Đăng Quy ${ }^{1}$, \\ Phạm Thùy Linh ${ }^{1}$, Nguyễn Tài Tuệ ${ }^{1}$, Lưu Việt Dũng ${ }^{1}$ \\ ${ }^{1}$ Truờng Đại học Khoa học Tụ nhiên, Đại học Quốc gia Hà Nội, 334 Nguyễn Trãi, Hà Nội, Việt Nam \\ ${ }^{2}$ Đảng ủy Khối các Co quan Trung uoong, 105B Quán Thánh, Hà Nội, Việt Nam \\ Nhận ngày 16 tháng 9 năm 2019 \\ Chỉnh sửa ngày 11 tháng 12 năm 2019; Chấp nhận đăng ngày 22 tháng 12 năm 2019
}

\begin{abstract}
Tóm tắt: Nghiên cứu này được thực hiện nhằm đề xuất bộ chỉ thị đánh giá tính hiệu quả của các đề tài, dự án khoa học và công nghệ. Tính hiệu quả của các đề tài, dự án được đánh giá dựa trên 03 hợp phần gồm 20 chỉ thị: 1) Hiệu quả về khoa học và công nghệ gồm 12 chỉ thị; 2) Hiệu quả về nguồn lực thực hiện gồm 04 chỉ thị; và 3) Hiệu quả về đào tạo gồm 04 chỉ thị. Các chỉ thị này được đánh giá định lượng theo thang điểm $0-1$, trong đó giá trị 0 thể hiện mức chưa hiệu quả và giá trị 1 thể hiện mức hiệu quả cao nhất. Các chỉ thị được đánh giá định lượng dựa vào số lượng kết quả, sản phẩm được tạo ra và so sánh giữa số lượng thực tế so với đăng kí trong hợp đồng/thuyết minh. Để kiểm nghiệm bộ chỉ thị, 08 đề tài, dự án thuộc Chương trình Khoa học và Công nghệ phục vụ phát triển bền vững vùng Tây Bắc (sau đây gọi là Chương trình Tây Bắc) thuộc các lĩnh vực tự nhiên, công nghệ, xã hội được lựa chọn từ 58 đề tài, dự án thuộc Chương trình Tây Bắc để đánh giá. Kết quả thống kê cho thấy, 8 đề tài, dự án đều có kết quả, sản phẩm đạt hoặc vượt mức so với hợp đồng/thuyết minh. Kết quả đánh giá định lượng cho thấy các đề tài thuộc nhóm xã hội $(06 \mathrm{X}, 12 \mathrm{X}$, $17 \mathrm{X})$ có giá trị dao động trong khoảng $0,55-0,75$; các đề tài thuộc nhóm tự nhiên và dự án sản xuất thử nghiệm $(02 \mathrm{C}, 06 \mathrm{C}, 08 \mathrm{C}, 03 \mathrm{~T}$, DA1) có giá trị dao động trong khoảng $0,55-0,72$. Theo đó, cả 8 đề tài, dự án khoa học và công nghệ đều được đánh giá là có hiệu quả, trong đó có 2 đề tài $(06 \mathrm{X}$ và $02 \mathrm{C})$ được đánh giá là có hiệu quả cao.
\end{abstract}

Tù khoá: Đánh giá tính hiệu quả, đề tài khoa học và công nghệ, chương trình Tây Bắc, chỉ thị.

\section{Mở đầu}

Hiệu quả (effectiveness) là khái niệm được sử dụng để đánh giá các nghiên cứu, đề tài, dự án, chương trình, tổ chức,... Đánh giá hiệu quả bao gồm việc đánh giá đầu ra, chất lượng, giá trị gia tăng, đổi mới, khả năng giảm chi phí [1] và thường tập trung vào việc đạt mục tiêu cuối cùng [2].
Đánh giá hiệu quả, tác động của nghiên cứu (research impact evaluation) và các đề tài, dự án, chương trình khoa học và công nghệ (KH\&CN) là cơ sở quan trọng giúp cho chính phủ xác định định hướng tăng cường tài trợ nghiên cứu [3]. Bên cạnh đó, đánh giá được hiệu quả, tác động của các nghiên cứu, đề tài, dự án, chương trình

\footnotetext{
*Tác giả liên hệ.

Địa chi email: nhuanmt@vnu.edu.vn

https://doi.org/10.25073/2588-1094/vnuees.4457
} 
KH\&CN có thể phục vụ cho công tác kiểm tra, quản lí, và chuyển giao những đóng góp của nghiên cứu tới các bên liên quan ở các cấp khác nhau (địa phương, quốc gia và cộng đồng quốc tế) $[4,5]$. Ngoài ra, việc đánh giá này còn là cơ sở để cải thiện chính sách, tăng cường dịch vụ và đóng góp tiến tới cải tiến chất lượng cho cộng đồng. Đối với các nhà quản lý chương trình $\mathrm{KH} \& \mathrm{CN}$, việc đánh giá này còn là công cụ hữu ích, quan trọng trong lập kế hoạch, thẩm định và thể hiện mục tiêu cần đạt là gì, quyết định phân bổ nguồn lực như thế nào, hoặc làm thế nào để điều chỉnh, sửa đổi và thiết kế lại chương trình và ước tính kết quả đầu ra dự kiến, hiệu quả tác động của chương trình.

Nhìn chung, việc đánh giá hiệu quả của các đề tài và dự án KH\&CN đã được nghiên cứu và thực hiện bài bản, đảm bảo tính chính xác và bộ chỉ thị là một trong những công cụ hữu hiệu cho nhiệm vụ này. Hiện nay, một số khung đánh giá được sử dụng phổ biến trong đánh giá đề tài, dự án $K H \& C N$ là: khung nghiên cứu xuất sắc của Vương quốc Anh (Research Excellence Framework - REF) [6,7]; khung nghiên cứu xuất sắc của Australia (Excellence in Research for Australia - ERA); khung Payback của Viện Nghiên cứu sức khoẻ Quốc gia, Canada (National Institute of Health Research - NIHR) $[4,8]$; khung chất lượng nghiên cứu của Australia (Australian Research Quality Framework - RQF) [9]; khung đóng góp nghiên cứu (Research Contribution Framework - RCF) [10]. Bên cạnh đó, các đề tài, dự án thuộc một số chương trình KH\&CN cũng được đề xuất và đánh giá tại Hàn Quốc [11,12], Hoa Kỳ [13,14] và Canada [15],...

Tại Việt Nam, vấn đề đánh giá hiệu quả hoạt động KH\&CN của nhiều tổ chức, đơn vị đã được thực hiện định kỳ hàng năm tại các đơn vị này; việc đánh giá và thẩm định các đề tài, dự án KH\&CN cũng đã được thực hiện thường xuyên, chủ yếu dựa vào thống kê, kiểm đếm các sản phẩm $\mathrm{KH} \& \mathrm{CN}$, sản phẩm đào tạo,... theo hợp đồng và đánh giá chất lượng của các kết quả, sản phẩm. Tuy nhiên, cho đến nay chưa có bộ chỉ số, chỉ thị và cách đánh giá định lượng tính hiệu quả của các đề tài, dự án $\mathrm{KH} \& \mathrm{CN}$ nào được đề xuất. Do vậy, để đánh giá hiệu quả của các nghiên cứu, đề tài, dự án KH\&CN cần xây dựng một bộ chỉ thị phù hợp trên cơ sở các bộ chỉ thị đã được áp dụng thành công trên thế giới nhằm đánh giá một cách chính xác và toàn diện các đề tài, dự án trong các chương trình $\mathrm{KH} \& \mathrm{CN}$ nói chung, giúp phân bổ nguồn lực hiệu quả và đưa ra những chính sách phù hợp cho các chương trình $\mathrm{KH} \& \mathrm{CN}$ giai đoạn tới.

Chương trình Tây Bắc giai đoạn 2013-2018 do Đại học Quốc gia Hà Nội chủ trì thực hiện có 55 đềं tài và 03 dự án sản xuất thử nghiệm. Các đề tài, dự án này được phân chia thành 04 nhóm chính: xã hội có ký hiệu đuôi là $X$ gồm 27 đề tài; công nghệ có kí hiệu đuôi là $C$ gồm 24 đề tài; tự nhiên có kí hiệu đuôi là $\mathrm{T}$ gồm 4 đề tài; và dự án sản xuất thử nghiệm có kí hiệu đuôi là DA gồm 03 dự án. Nghiên cứu này được thực hiện nhằm đề xuất bộ chỉ thị đánh giá hiệu quả của các đề tài, dự án $\mathrm{KH} \& \mathrm{CN}$ và thử nghiệm bộ chỉ thị này thông qua đánh giá hiệu quả của 08 đề tài thuộc Chương trình Tây Bắc.

\section{Phương pháp nghiên cứu}

- Phương pháp xây dựng bộ chỉ thị đánh giá tính hiệu quả của đề tài, dự án $K H \& C N$ : Bộ chỉ thị này được xây dựng dựa trên việc phân tích, kế thừa, tổng hợp các chỉ thị đánh giá hiệu quả của các đề tài, dự án $\mathrm{KH} \& \mathrm{CN}$ trên thế giới và tại Việt Nam, đồng thời điều chỉnh cho phù hợp với kết quả, sản phẩm của các đề tài, dự án $\mathrm{KH} \& \mathrm{CN}$ thuộc Chương trình Tây Bắc.

- Phương pháp lựa chọn đề tài, dự án để đánh giá: Các đề tài, dự án được lựa chọn dựa trên tiến độ nghiệm thu (chỉ lựa chọn các đề tài, dự án thuộc Chương trình Tây Bắc đã được nghiệm thu tính đến thời điểm đánh giá); sự cân đối giữa đề tài thuộc các nhóm xã hội, công nghệ, tự nhiên, và dự án sản xuất thử nghiệm; thông tin, dữ liệu đầu vào phục vụ đánh giá theo bộ chỉ thị. Theo đó, 08 đề tài, dự án thuộc Chương trình Tây Bắc (Bảng 1) được lựa chọn ngẫu nhiên phục vụ đánh giá, trong đó bao gồm 03 đề tài thuộc nhóm xã hội, 03 đề tài thuộc nhóm công nghệ, 01 đề tài thuộc nhóm tự nhiên, và 01 dự án sản xuất thử nghiệm. 
- Phương pháp đánh giá hiệu quả của các đề tài/dự án: Hiệu quả của các đề tài, dự án thuộc Chương trình Tây Bắc được đánh giá dựa vào bộ chỉ thị đã được xây dựng. Các thông tin đầu vào để đánh giá tính hiệu quả của đề tài, dự án KH\&CN theo bộ chỉ thị được thu thập, thống kê, chắt lọc từ các thuyết minh, báo cáo tổng hợp, báo cáo thống kê của 08 đề tài, dự án KH\&CN thuộc Chương trình Tây Bắc (Bảng 1).

Việc đánh giá đề tài, dự án $\mathrm{KH \& CN}$ không chỉ dựa vào việc triển khai so với đăng ký ban đầu mà còn phải dựa trên số lượng các kết quả, sản phẩm được tạo ra. Do đó, các chỉ thị đánh giá được tính toán theo hai cách thức: i) So sánh giữa số lượng thực tế với số lượng theo hợp đồng (với các kết quả, sản phẩm có trong hợp đồng giữa Chương trình Tây Bắc với các đề tài, dự án); và ii) So sánh số lượng các kết quả, sản phẩm giữa các đề tài, dự án thuộc cùng một chương trình KH\&CN. Đối với chỉ thị hiệu quả kinh phí được tính bằng tỉ số giữa tổng kinh phí được cấp trên số lượng sản phẩm $\mathrm{KH \& CN}$ và sản phẩm đào tạo (thạc sĩ, nghiên cứu sinh) được tạo thành. Theo đó, kinh phí chi cho mỗi sản phẩm $K H \& C N$ và đào tạo càng thấp thì tính hiệu quả càng cao. Ngoài ra, đối với các đề tài, dự án KH\&CN không có một loại sản phẩm $\mathrm{KH} \& \mathrm{CN}$ nào đó (theo hợp đồng) thì không tính điểm đối với chỉ thị đó đó.

Kết quả thu được từ các chỉ tiêu đánh giá đơn lẻ có giá trị thứ nguyên khác nhau, do đó chúng cần được chuẩn hóa về thành đại lượng không có thứ nguyên để tính toán và đánh giá. Các chỉ thị này được chuẩn hóa về giá trị trong khoảng từ 0 đến 1 , trong đó giá trị 0 thể hiện mức chưa hiệu quả và giá trị 1 thể hiện mức hiệu quả cao nhất (tuyệt đối) theo hai phương pháp: i) Chuẩn hóa theo phương pháp min - max đối với các chỉ thị có giá trị định lượng (phương trình 1) [16,17]; ii) Quy đổi theo thang điểm từ 0 đến 1 đối với các chỉ thị có giá trị bán định lượng (so sánh giữa số lượng thực tế với số lượng theo hợp đồng):

Bảng 1. Danh mục 08 đề tài, dự án KH\&CN thuộc Chương trình Tây Bắc được lựa chọn để đánh giá tính hiệu quả

\begin{tabular}{|c|c|c|}
\hline STT & Ký hiệu & Tên đề tài, dự án \\
\hline 1 & $06 \mathrm{X}$ & Nghiên cứu xây dựng chuỗi cung ứng sản phẩm nông lâm đặc sản xuất khẩu. \\
\hline 2 & $12 \mathrm{X}$ & Nghiên cứu biến đổi xã hội vùng Tây Bắc phục vụ xây dựng mô hình phát triển bền vững. \\
\hline 3 & $17 \mathrm{X}$ & $\begin{array}{l}\text { Nghiên cứu giải pháp nâng cao hiệu quả vận động đồng bào các dân tộc khu vực biên giới } \\
\text { Tây Bắc trong bảo vệ chủ quyền, an ninh biên giới quốc gia. }\end{array}$ \\
\hline 4 & $02 \mathrm{C}$ & $\begin{array}{l}\text { Nghiên cứu, áp dụng công nghệ tích hợp địa môi trường - địa sinh thái nhằm ngăn ngừa, } \\
\text { xử lý ô nhiễm môi trường nước vùng khai thác, chế biến khoáng sản. }\end{array}$ \\
\hline 5 & $06 \mathrm{C}$ & $\begin{array}{l}\text { Úng dụng và triển khai hệ thống phần mềm tích hợp và kết nối các thiết bị điện tử y sinh } \\
\text { và mạng truyền thông hỗ trợ theo dõi sức khoẻ và dịch tễ cộng đồng. }\end{array}$ \\
\hline 6 & 08C & $\begin{array}{l}\text { Nghiên cứu ứng dụng một số vật liệu tiên tiến, thân thiện môi trường trong canh tác nông, } \\
\text { lâm nghiệp. }\end{array}$ \\
\hline 7 & $03 \mathrm{~T}$ & $\begin{array}{l}\text { Đánh giá tài nguyên đất nông nghiệp, đề xuất mô hình sử dụng đất với cơ cấu cây trồng có } \\
\text { hiệu quả kinh tề và bền vững về môi trường. }\end{array}$ \\
\hline 8 & DA1 & Thử nghiệm nhân trồng, phát triển cây Mắc ca. \\
\hline
\end{tabular}


Bảng 2. Bộ chị thị đánh giá hiệu quả của các đề tài, dự án KH\&CN

\begin{tabular}{|c|c|c|c|}
\hline STT & Chì thị & Ký hiệu & Nguồn tham khảo \\
\hline I. & Hiệu quả về KH\&CN & & \\
\hline 1. & $\begin{array}{l}\text { Số lượng bài báo đã công bố trên các tạp chí thuộc hệ } \\
\text { thống ISI/Scopus. }\end{array}$ & HQ1 & $\begin{array}{l}\text { ERA, NIHR, RQF, Hàn Quốc; } \\
{[3,18,19] \text {. }}\end{array}$ \\
\hline 2. & $\begin{array}{l}\text { Số lượng bài báo đã công bố trên các tạp chí quốc tế } \\
\text { không thuộc hệ thống ISI/Scopus. }\end{array}$ & HQ2 & $\begin{array}{l}\text { ERA, NIHR, RQF, Hàn Quốc; } \\
{[3,18,19] \text {. }}\end{array}$ \\
\hline 3. & $\begin{array}{l}\text { Số bài báo đã công bố trên các tạp chí uy tín trong } \\
\text { nước. }\end{array}$ & HQ3 & $\begin{array}{l}\text { ERA, NIHR, RQF, Hàn Quốc; } \\
{[3,18,19] \text {. }}\end{array}$ \\
\hline 4. & Số bài đã đăng trong kỷ yếu hội thảo quốc tế. & HQ4 & RQF, SEP; [3,18,19]. \\
\hline 5. & Số bài đã đăng trong kỷ yếu hội thảo quốc gia. & HQ5 & \\
\hline 6. & Số lượng sách được xuất bản. & HQ6 & ERA; $[3,18,19]$. \\
\hline 7. & $\begin{array}{l}\text { Số lượng sở hữu trí tuệ (bằng sáng chế, giải pháp hữu } \\
\text { ích...) đã đăng ký hoặc được cấp. }\end{array}$ & HQ7 & NIHR, Hàn Quốc, Canada. \\
\hline 8. & $\begin{array}{l}\text { Số sản phẩm KH\&CN được chuyển giao cho các đối } \\
\text { tượng sử dụng. }\end{array}$ & HQ8 & Chương trình $\mathrm{KC} 04$. \\
\hline 9. & Số sản phẩm KH\&CN được thương mại hóa. & HQ9 & ERA, Canada. \\
\hline 10. & Số sản phẩm KH\&CN được áp dụng trong thực tế. & HQ10 & Canada. \\
\hline 11. & $\begin{array}{l}\text { Số sản phẩm KH\&CN tạo ra tri thức khoa học mới } \\
\text { và có giá trị. }\end{array}$ & HQ11 & SEP, Canada, REF. \\
\hline 12. & $\begin{array}{l}\text { Số sản phẩm KH\&CN được sử dụng để hoạch định } \\
\text { chính sách, quy hoạch phát triền kinh tế - xã hội. }\end{array}$ & HQ12 & \\
\hline II. & Hiệu quả về nguồn lực thực hiện & & \\
\hline 13. & $\begin{array}{l}\text { Tổng kinh phí được cấp cho các đề tài, dự án (triệu } \\
\text { đồng). }\end{array}$ & HQ13 & Hàn Quốc, Mỹ. \\
\hline 14. & Số lượng tổ chức phối hợp thực hiện đề tài, dự án. & HQ14 & \\
\hline 15. & $\begin{array}{l}\text { Số cán bộ tham gia thực hiện các đề tài, dự án có } \\
\text { trình độ tiến sĩ trở lên. }\end{array}$ & HQ15 & Hàn Quốc, Mỹ. \\
\hline 16. & $\begin{array}{l}\text { Số cán bộ có trình độ thạc sĩ, cử nhân, kỹ sư tham gia } \\
\text { đề tài, dự án. }\end{array}$ & HQ16 & Hàn Quốc, Mỹ. \\
\hline III. & Hiệu quả về đào tạo & & \\
\hline 17. & Số thạc sỹ đã đào tạo. & HQ17 & NIHR, Hàn Quốc. \\
\hline 18. & Số nghiên cứu sinh được hỗ trợ. & HQ18 & NIHR, SEP, Hàn Quốc \\
\hline 19. & $\begin{array}{l}\text { Số lượng cán bộ địa phương và người dân được đào } \\
\text { tạo, tập huấn nâng cao trình độ. }\end{array}$ & HQ19 & \\
\hline 20. & Số lượng hội nghị và hội thảo khoa học đã tổ chức. & HQ20 & \\
\hline
\end{tabular}

Ghi chú: NIHR - National Institute of Health Research (Khung Payback của Viện Nghiên cứu sức khoẻ Quốc gia, Canada), $R E F$ - Research Excellence Framework (Khung nghiên cúu xuát sắc của Vurong quồc Anh), RQF - Australian Research Quality Framework (Khung chất luợng nghiên cúu của Australia), ERA - Excellence in Research for Australia (Khung nghiên cứu xuất sắc của Australia).

$$
\mathrm{x}_{\mathrm{ij}}=\frac{\mathrm{X}_{\mathrm{ij}}-\mathrm{MinX}_{\mathrm{ij}}}{\operatorname{MaxX}_{\mathrm{ij}}-\operatorname{MinX}_{\mathrm{ij}}}
$$

Trong đó: $x_{i j}$ là giá trị chuẩn hóa ở chỉ thị $i$ của đối tuợng $j ; X_{i j}$ là giá trị thục của chỉ thị $i$ của đối tượng j; các giá trị Max và Min là giá trị lớn nhất và nhỏ nhất của tù̀ng chỉ thị.

Giá trị tổng hợp đánh giá định lượng tính hiệu quả của các đề tài, dự án $\mathrm{KH} \& \mathrm{CN}$ được tính theo

phương pháp trung bình cộng giá trị đạt được của tổng các chỉ thị và được phân thành ba cấp độ như sau: i) Chưa hiệu quả $(0$ - 0,33$)$; ii) Hiệu quả $(0,34$ - 0,67); iii) Hiệu quả cao $(0,68$ - 1,00).

- Phương pháp phỏng vấn sâu: Được thực hiện nhằm đề xuất các giải pháp nâng cao hiệu quả của các đề tài, dự án thuộc Chương trình Tây Bắc. Phương pháp này được thực hiện đối với 
các cán bộ chuyên trách cấp Sở tại 14 tỉnh thuộc vùng Tây Bắc (Hà Giang, Lào Cai, Yên Bái, Lai Châu, Điện Biên, Sơn La, Hoà Bình, Cao Bằng, Bắc Kạn, Lạng Sơn, Phú Thọ, Tuyên Quang, Thanh Hóa, Nghệ An), các chuyên gia và cán bộ tham gia trực tiếp các đề tài, dự án thuộc chương trình Tây Bắc.

\section{Kết quả và thảo luận}

\subsection{Xây dựng bộ chỉ thị đánh giá hiệu quả của đề tài, dụ án}

Bộ chỉ thị đánh giá hiệu quả của đề tài, dự án KH\&CN được đề xuất bao gồm 20 chỉ thị thuộc 3 hợp phần: i) Hiệu quả về KH\&CN gồm 12 chỉ thị; ii) Hiệu quả về nguồn lực thực hiện gồm 04 chỉ thị; và iii) Hiệu quả về đào tạo gồm 04 chỉ thị (Bảng 2).

\subsection{Kết quả, sản phẩm của các đề tài, dụ án}

Thống kê số lượng và tỷ lệ các kết quả, sản phẩm của các đề tài, dự án được trình bày trong Bảng 3 và Bảng 4 . Tỷ lệ các kết quả, sản phẩm của các đề tài, dự án được tính dựa trên tỷ số giữa số lượng các kết quả, sản phẩm và số lượng các đề tài, dự án có các kết quả, sản phẩm này. Bảng 3 cho thấy tất cả các đề tài, dự án đều có kết quả, sản phẩm đạt hoặc vượt mức so với hợp đồng.

Bảng 3. Thống kê kết quả, sản phẩm của 08 đề tài, dự án KH\&CN thuộc Chương trình Tây Bắc đã lựa chọn theo hợp đồng và đạt được trên thực tế

\begin{tabular}{|c|c|c|c|c|c|c|c|c|c|c|c|c|c|c|c|c|}
\hline \multirow{2}{*}{ Chỉ thị } & \multicolumn{2}{|c|}{$06 \mathrm{X}$} & \multicolumn{2}{|c|}{$12 \mathrm{X}$} & \multicolumn{2}{|c|}{$17 \mathrm{X}$} & \multicolumn{2}{|c|}{$02 \mathrm{C}$} & \multicolumn{2}{|c|}{$06 \mathrm{C}$} & \multicolumn{2}{|c|}{$08 \mathrm{C}$} & \multicolumn{2}{|c|}{$03 \mathrm{~T}$} & \multicolumn{2}{|c|}{ DA1 } \\
\hline & $\mathrm{HĐ}$ & TT & $\mathrm{HĐ}$ & TT & $\mathrm{H}$ & TT & $\mathrm{HĐ}$ & TT & $\mathrm{H}$ & TT & HĐ & TT & HĐ & TT & HĐ & TT \\
\hline \multicolumn{17}{|c|}{ I. Hiệu quả về KH\&CN } \\
\hline HQ1 & 0 & 0 & 0 & 0 & 0 & 0 & 1 & 4 & 0 & 2 & 0 & 0 & 1 & 2 & 0 & 0 \\
\hline HQ2 & 0 & 0 & 0 & 0 & 0 & 0 & 0 & 1 & 0 & 1 & 0 & 0 & 0 & 0 & 0 & 0 \\
\hline HQ3 & 5 & 8 & 5 & 5 & 5 & 6 & 2 & 4 & 2 & 3 & 5 & 5 & 3 & 4 & 1 & 1 \\
\hline HQ4 & 0 & 0 & 0 & 0 & 0 & 0 & 2 & 3 & 0 & 0 & 0 & 0 & 0 & 0 & 0 & 0 \\
\hline HQ5 & 0 & 0 & 0 & 0 & 0 & 0 & 0 & 0 & 0 & 0 & 0 & 0 & 0 & 0 & 0 & 0 \\
\hline HQ6 & 0 & 0 & 1 & 1 & 1 & 3 & 0 & 0 & 0 & 0 & 0 & 0 & 0 & 0 & 0 & 0 \\
\hline HQ7 & 0 & 0 & 0 & 0 & 0 & 0 & 0 & 1 & 1 & 1 & 1 & 1 & 0 & 0 & 0 & 0 \\
\hline HQ8 & 2 & 3 & 1 & 2 & 1 & 4 & 5 & 7 & 2 & 3 & 4 & 5 & 2 & 7 & 1 & 5 \\
\hline HQ9 & 0 & 0 & 0 & 0 & 0 & 0 & 0 & 0 & 1 & 1 & 0 & 0 & 0 & 0 & 0 & 0 \\
\hline HQ10 & 0 & 0 & 0 & 0 & 0 & 2 & 1 & 1 & 1 & 1 & 0 & 0 & 0 & 0 & 1 & 1 \\
\hline HQ11 & - & 1 & - & 1 & 0 & 3 & - & 3 & - & 0 & - & 4 & - & 0 & - & 5 \\
\hline HQ12 & - & 3 & - & 3 & - & 1 & - & 1 & - & 0 & - & 1 & - & 2 & - & 1 \\
\hline \multicolumn{17}{|c|}{ II. Hiệu quả về nguồn lực thực hiện } \\
\hline HQ13 & \multicolumn{2}{|c|}{2.500} & \multicolumn{2}{|c|}{2.500} & \multicolumn{2}{|c|}{3.000} & \multicolumn{2}{|c|}{4.800} & \multicolumn{2}{|c|}{5.000} & \multicolumn{2}{|c|}{4.800} & \multicolumn{2}{|c|}{4.500} & \multicolumn{2}{|c|}{4.200} \\
\hline HQ14 & 8 & 8 & 1 & 1 & 1 & 1 & 2 & 2 & 6 & 6 & 5 & 5 & 3 & 3 & 4 & 7 \\
\hline HQ15 & 10 & 10 & 3 & 3 & 6 & 6 & 10 & 10 & 6 & 6 & 6 & 5 & 3 & 3 & 9 & 9 \\
\hline HQ16 & 0 & 0 & 6 & 6 & 3 & 3 & 0 & 1 & 4 & 4 & 1 & 2 & 7 & 7 & 1 & 1 \\
\hline \multicolumn{17}{|c|}{ III. Hiệu quả về đào tạo } \\
\hline HQ17 & 2 & 3 & 2 & 2 & 2 & 2 & 2 & 5 & 2 & 2 & 2 & 2 & 3 & 4 & 0 & 0 \\
\hline HQ18 & 1 & 1 & 1 & 1 & 1 & 1 & 1 & 1 & 1 & 1 & 1 & 1 & 1 & 1 & 0 & 0 \\
\hline HQ19 & 0 & 0 & 0 & 0 & 0 & 0 & 0 & 0 & 453 & 453 & 0 & 0 & 0 & 0 & 900 & 900 \\
\hline HQ20 & 3 & 3 & 3 & 3 & 1 & 1 & 2 & 2 & 0 & 1 & 5 & 5 & 2 & 2 & 0 & 2 \\
\hline
\end{tabular}

Ghi chú: $H Đ$ - Kết quả, sản phẩm KH\&CN theo hợp đồng; TT - Kết quả, sản phẩm KH\&CN theo thực tế. 
Bảng 4. Tỷ lệ kết quả, sản phẩm của 08 đề tài, dự án KH\&CN thuộc Chương trình Tây Bắc đã lựa chọn

\begin{tabular}{|c|c|c|}
\hline Chỉ thị & Ký hiệu & $\begin{array}{l}\text { Tỷ lệ kết quả, } \\
\text { sản phẩm, kinh } \\
\text { phí/đề tài, dự án }\end{array}$ \\
\hline \multicolumn{3}{|l|}{ I. Hiệu quả về khoa học - công nghệ } \\
\hline Số lượng bài báo đã công bố trên các tạp chí thuộc hệ thống ISI/Scopus. & HQ1 & 1,00 \\
\hline $\begin{array}{l}\text { Số lượng bài báo đã công bố trên các tạp chí quốc tế không thuộc hệ thống } \\
\text { ISI/Scopus. }\end{array}$ & HQ2 & 0,25 \\
\hline Số bài báo đã công bố trên các tạp chí uy tín trong nước. & HQ3 & 4,50 \\
\hline Số bài đã đăng trong kỷ yếu hội thảo quốc tế. & HQ4 & 0,38 \\
\hline Số bài đã đăng trong kỷ yếu hội thảo trong nước. & HQ5 & 0 \\
\hline Số lượng sách được xuất bản. & HQ6 & 0,50 \\
\hline $\begin{array}{l}\text { Số lượng sở hữu trí tuệ (bằng sáng chế, giải pháp hữu ích...) đã đăng ký hoặc } \\
\text { được cấp. }\end{array}$ & HQ7 & 0,38 \\
\hline Số sản phẩm KH\&CN được chuyển giao cho các đối tượng sử dụng. & HQ8 & 4,50 \\
\hline Số sản phẩm KH\&CN được thương mại hóa. & HQ9 & 0,13 \\
\hline Số sản phẩm KH\&CN được áp dụng trong thực tế. & HQ10 & 0,63 \\
\hline Số sản phẩm KH\&CN tạo ra tri thức khoa học mới và có giá trị. & HQ11 & 2,13 \\
\hline $\begin{array}{l}\text { Số sản phẩm KH\&CN được sử dụng để hoạch định chính sách, quy hoạch } \\
\text { phát triển kinh tế - xã hội. }\end{array}$ & HQ12 & 1,50 \\
\hline \multicolumn{3}{|l|}{ II. Hiệu quả về nguồn lực thực hiện } \\
\hline Tổng kinh phí được cấp cho các đề tài, dự án (triệu đồng). & HQ13 & 3912,5 \\
\hline Số lượng tổ chức phối hợp thực hiện đề tài, dự án. & HQ14 & 4,13 \\
\hline Số cán bộ tham gia thực hiện các đề tài, dự án có trình độ tiến sĩ trở lên. & HQ15 & 6,50 \\
\hline Số cán bộ có trình độ thạc sĩ, cử nhân, kỹ sư tham gia đề tài, dự án. & HQ16 & 3,00 \\
\hline \multicolumn{3}{|l|}{ III. Hiệu quả về đào tạo } \\
\hline Số thạc sỹ đã đào tạo. & HQ17 & 2,50 \\
\hline Số nghiên cứu sinh được hỗ trợ. & HQ18 & 0,88 \\
\hline $\begin{array}{l}\text { Số lượng cán bộ địa phương và người dân được đào tạo, tập huấn nâng cao } \\
\text { trình độ. }\end{array}$ & HQ19 & 169 \\
\hline Số lượng hội nghị và hội thảo khoa học đã tổ chức. & HQ20 & 2,38 \\
\hline
\end{tabular}

Đối với sản phẩm KH\&CN, các chỉ thị số bài báo đã công bố trên các tạp chí quốc tế không thuộc hệ thống ISI/Scopus (HQ2), số bài đăng trên kỷ yếu hội thảo quốc tế (HQ4) và trong nước (HQ5), số lượng sở hữu trí tuệ đã đăng ký hoặc được cấp
(HQ7), số sản phẩm KH\&CN được thương mại hóa (HQ9) thấp hơn so với các chỉ thị khác (Bảng 4). Trong số 08 đề tài, dự án được đánh giá, chỉ có 01 đề tài có bài đăng trên kỷ yếu hội thảo quốc tế và 01 đề tài có sản phẩm được thương mại hóa 
(Bảng 3). Tuy nhiên, các chỉ tiêu số bài báo đã công bố trên các tạp chí uy tín trong nước (HQ3), số sản phẩm KH\&CN được chuyển giao cho các đối tượng sử dụng (HQ8) tương đối cao. Các đề tài thuộc nhóm tự nhiên $(02 \mathrm{C}, 06 \mathrm{C}, 08 \mathrm{C}, 03 \mathrm{~T})$ có các chỉ tiêu tỷ lệ bài báo đăng trên tạp chí quốc tế (HQ1, HQ2), số sản phẩm KH\&CN được chuyển giao cho các đối tượng sử dụng (HQ8) cao hơn các đề tài thuộc nhóm xã hội (06X, 12X, $17 \mathrm{X})$. Ngược lại, các đề tài thuộc nhóm xã hội có chỉ thị số bài báo trong nước (HQ3) cao hơn so với các nhóm đề tài còn lại (Bảng 3).

Đối với chỉ thị hiệu quả về nguồn lực thực hiện và đào tạo, không có sự khác biệt lớn giữa các nhóm đề tài, dự án KH\&CN. Tuy nhiên, dự án (DA1) không có sản phẩm đào tạo (HQ17, HQ18) nhưng có số lượng cán bộ địa phương và người dân được đào tạo, tập huấn nâng cao trình độ (HQ19) cao nhất với 900 người (Bảng 3), đây cũng là đặc thù riêng của dự án này.

\section{3. Đánh giá hiệu quả của các đề tài, dụ án}

Kết quả đánh giá hiệu quả của các đề tài, dự án theo các khía cạnh $\mathrm{KH \& CN}$, nguồn lực thực hiện và đào tạo được trình bày trong Bảng 5 . Nhìn chung, các đề tài, dự án $K H \& C N$ thuộc nhóm tự nhiên và dự án có hiệu quả về sản phẩm $\mathrm{KH} \& \mathrm{CN}$ được chuyển giao cho các đối tượng sử dụng (HQ8) cao hơn so với các đề tài, dự án KH\&CN nhóm xã hội. Tuy nhiên, các đề tài, dự án KH\&CN nhóm xã hội có hiệu quả về sản phẩm KH\&CN được sử dụng để hoạch định chính sách, quy hoạch phát triển kinh tế - xã hội (HQ12) cao hơn so với các đề tài, dự án KH\&CN thuộc nhóm tự nhiên. Bên cạnh đó, trừ đề tài $02 \mathrm{C}$, các các đề tài, dự án KH\&CN thuộc nhóm tự nhiên có hiệu quả về kinh phí được cấp (triệu đồng) để tạo ra một sản phẩm $K H \& C N$ và đào tạo (HQ13) thấp hơn so với các đề tài, dự án KH\&CN thuộc nhóm xã hội. Điều này có thể do kinh phí để tạo ra các sản phẩm $\mathrm{KH} \& \mathrm{CN}$ nói chung và sản phẩm ứng dụng trên thực tế của đề tài thuộc nhóm tự nhiên cao hơn so với nhóm xã hội. Các chỉ thi khác có giá trị thay đổi tùy theo kết quả, sản phẩm của từng đề tài, dự án.
Về $\mathrm{KH} \& \mathrm{CN}$, đề tài có hiệu quả cao nhất và thấp nhất tương ứng là $02 \mathrm{C}$ và $12 \mathrm{X}$ (Bảng 5$)$. Về nguồn lực thực hiện, đề tài có hiệu quả cao nhất và thấp nhất tương ứng là $06 \mathrm{X}$ và $06 \mathrm{C}$. Về đào tạo, đề tài có hiệu quả cao nhất và thấp nhất tương ứng là $02 \mathrm{C}$ và $17 \mathrm{X}$. Đánh giá hiệu quả của 3 khía cạnh này, đề tài có hiệu quả cao nhất là $06 \mathrm{X}$ và thấp nhất là $12 \mathrm{X}$ và $06 \mathrm{C}$ (Bảng 5$)$. Kết quả đánh giá cũng cho thấy hiệu quả của đề tài nhóm xã hội dao động trong khoảng 0,55 - 0,75; đề tài thuộc nhóm tự nhiên và dự án dao động trong khoảng $0,55-0,72$. Kết quả này cho thấy bộ chỉ thị này có thể được sử dụng đối với đánh giá các đề tài, dự án KH\&CN ở các lĩnh vực khác nhau. Tuy nhiên, đây mới là kết quả đánh giá bước đầu về hiệu quả của một số đề tài, dự án đã triển khai thuộc Chương trình Tây Bắc. Cần có những nghiên cứu, đề xuất các trọng số cho phù hợp, ví dụ nâng cao trọng số về số sản phẩm $\mathrm{KH} \& \mathrm{CN}$ được sử dụng để hoạch định chính sách, quy hoạch phát triển kinh tế - xã hội đối với đề tài thuộc nhóm xã hội, số sản phẩm chuyển giao, ứng dụng trong thực tế,... đối với đề tài thuộc nhóm tự nhiên và dự án sản xuất thử nghiệm. Bên cạnh đó, cần có thêm đánh giá của các bên liên quan, nhất là đối tượng sử dụng, thụ hưởng trực tiếp từ kết quả, sản phẩm từ các các đề tài, dự án $\mathrm{KH} \& \mathrm{CN}$ để kết quả đánh giá được thiết thực và phù hợp hơn.

\subsection{Các giải pháp nâng cao hiệu quả của các đề tài, $d u ̛$ án}

Kết quả thống kê các kết quả, sản phẩm và đánh giá hiệu quả của đề tài, dự án cho thấy sự cần thiết của việc áp dụng các giải pháp nhằm nâng cao hiệu quả của các đề tài, dự án, đặc biệt với các chỉ thị HQ2, HQ4, HQ5, HQ7, HQ9. Kết hợp với kết quả phỏng vấn sâu, các nhóm giải pháp cần triển khai nhằm nâng cao hiệu quả của các đề tài, dự án thuộc Chương trình Tây Bắc như sau:

\section{a) Nhóm giải pháp về $\mathrm{KH} \& \mathrm{CN}$ :}

Hiệu quả của các các đề tài, dự án KH\&CN phụ thuộc rất lớn vào tính mới, tính độc đáo, tính thiết thực, chất lượng, khả năng áp dụng/ứng dụng của các kết quả, sản phẩm: 
Bảng 5. Kết quả đánh giá hiệu quả của 08 đề tài, dự án KH\&CN thuộc Chương trình Tây Bắc đã lựa chọn

\begin{tabular}{|c|c|c|c|c|c|c|c|c|}
\hline Chỉ thị & $06 \mathrm{X}$ & $12 \mathrm{X}$ & $17 X$ & $02 \mathrm{C}$ & $06 \mathrm{C}$ & 08C & $03 \mathrm{~T}$ & DA1 \\
\hline \multicolumn{9}{|c|}{ I. Hiệu quả về KH\&CN } \\
\hline HQ1 & - & - & - & 1,00 & 0,75 & - & 0,75 & - \\
\hline HQ2 & - & - & - & 1,00 & 1,00 & - & - & - \\
\hline HQ3 & 1,00 & 0,54 & 0,86 & 0,71 & 0,64 & 0,54 & 0,71 & 0,25 \\
\hline HQ4 & - & - & - & 1,00 & - & - & - & - \\
\hline HQ5 & - & - & - & - & - & - & - & - \\
\hline HQ6 & - & 0,42 & 1,00 & - & - & - & - & - \\
\hline HQ7 & - & - & - & 1,00 & 0,75 & 0,75 & - & - \\
\hline HQ8 & 0,69 & 0,63 & 0,75 & 0,94 & 0,69 & 0,81 & 0,94 & 0,81 \\
\hline HQ9 & - & - & - & - & 1,00 & - & - & - \\
\hline HQ10 & - & - & 1,00 & 0,50 & 0,50 & - & - & 0,50 \\
\hline HQ11 & 0,20 & 0,20 & 0,60 & 0,60 & 0 & 0,80 & 0 & 1,00 \\
\hline HQ12 & 1,00 & 1,00 & 0,33 & 0,33 & 0 & 0,33 & 0,67 & 0,33 \\
\hline Trung bình & 0,72 & 0,56 & 0,76 & 0,79 & 0,59 & 0,65 & 0,61 & 0,58 \\
\hline \multicolumn{9}{|c|}{ II. Hiệu quả về nguồn lực thực hiện } \\
\hline HQ13 & 1,00 & 0,83 & 0,98 & 0,89 & 0 & 0,40 & 0,54 & 0,05 \\
\hline HQ14 & 0,75 & 0,31 & 0,31 & 0,38 & 0,63 & 0,56 & 0,44 & 0,94 \\
\hline HQ15 & 0,75 & 0,25 & 0,46 & 0,75 & 0,46 & 0,14 & 0,25 & 0,68 \\
\hline HQ16 & - & 0,68 & 0,46 & 0,57 & 0,54 & 0,64 & 0,75 & 0,32 \\
\hline Trung bình & 0,83 & 0,52 & 0,55 & 0,65 & 0,41 & 0,44 & 0,49 & 0,50 \\
\hline \multicolumn{9}{|c|}{ III. Hiệu quả về đào tạo } \\
\hline HQ17 & 0,80 & 0,45 & 0,45 & 1,00 & 0,45 & 0,45 & 0,90 & - \\
\hline HQ18 & 0,75 & 0,75 & 0,75 & 0,75 & 0,75 & 0,75 & 0,75 & - \\
\hline HQ19 & - & - & - & - & 0,75 & - & - & 0,75 \\
\hline HQ20 & 0,55 & 0,55 & 0,35 & 0,45 & 0,60 & 0,75 & 0,45 & 0,70 \\
\hline Trung bình & 0,70 & 0,58 & 0,52 & 0,73 & 0,64 & 0,65 & 0,70 & 0,73 \\
\hline $\begin{array}{c}\text { Điểm trung } \\
\text { bình }\end{array}$ & 0,75 & 0,55 & 0,61 & 0,72 & 0,55 & 0,58 & 0,60 & 0,60 \\
\hline
\end{tabular}


- Việc lựa chọn các đề tài, dự án cần dựa trên khả năng tạo ra các kết quả, sản phẩm mới: sản phẩm hàng hóa mới, giống cây trồng vật nuôi mới, vật liệu mới, thiết bị máy móc mới, dây chuyền công nghệ mới, quy trình kỹ thuật/công nghệ mới, mô hình mới, phần mềm máy tính mới, phương pháp mới, quy luật mới, giải pháp mới, khuyến nghị mới, thông tin mới, cơ sở dữ liệu mới,...

- Ưu tiên lựa chọn các đề tài, dự án xuất phát từ nhu cầu/đặt hàng của các địa phương và các ý tưởng mới, thiết thực;

- Chú trọng quản trị $\mathrm{KH} \& \mathrm{CN}$ theo sản phẩm/kết quả cuối cùng (phương pháp mới, quy trình mới, số liệu và dữ liệu mới, phát hiện mới, đề xuất mới hữu dụng,...) và quản trị sáng tạo.

b) Nhóm giải pháp về sự liên kết, phối hợp giữa các bên liên quan:

- Tăng cường sự liên kết, phối hợp giữa các chương trình $\mathrm{KH} \& \mathrm{CN}$ nói chung và Chương trình Tây Bắc nói riêng, với các địa phương (đầu mối chính là Sở KH\&CN) trong các khâu đề xuất, lựa chọn, thực hiện, đánh giá các đề tài, dự án;

- Có đại diện của đơn vị đặt hàng (UBND tỉnh, các sở của tỉnh như KH\&CN, Nội vụ, Giáo dục và Đào tạo, Nông nghiệp và Phát triển nông thôn, Công thương, Văn hoá, Thể thao, Du lịch, ..., doanh nghiệp) tham gia đề xuất, xét duyệt, lựa chọn, triển khai, đánh giá các đề tài, dự án liên quan đến địa phương triển khai;

- Huy động các cán bộ, nhà khoa học địa phương tham gia ngay từ khâu đề xuất, xây dựng thuyết minh, tổ chức thực hiện, nghiệm thu và sử dụng kết quả của đề tài, dự án;

- Tăng cường sự liên kết, phối hợp giữa đề tài, dự án với sở $K H \& C N$ và đơn vị liên quan trực tiếp đến sử dụng kết quả của đề tài, dự án (ví dụ: Sở Nội vụ, Sở Kế hoạch và Đầu tư, Sở Văn hóa, Thể thao và $\mathrm{Du}$ lịch, Sở Giáo dục và Đào tạo,...) trong quá trình xây dựng, triển khai và sử dụng/ứng dụng kết quả;

- Thường xuyên gửi các thông tin cập nhật về tình hình thực hiện đề tài, dự án cho các bên liên quan nhằm giải quyết kịp thời các vấn đề khó khăn của đề tài khi thực hiện cũng như để cơ quan quản lý nắm được tình hình thực hiện đề tài, có quyết định điều chỉnh kịp thời trong các trường hợp đề tài, dự án triển khai nhưng không thực sự phù hợp;

- Cơ quan quản lý và đối tượng sử dụng cuối cùng phải khảo sát thực tế, đánh giá tại hiện trường nhằm đánh giá kết quả một cách khách quan, chính xác nhất, làm cơ sở để sử dụng/ứng dụng các kết quả, sản phẩm sau khi đề tài, dự án hoàn thành.

- Tăng cường sự tham gia của các đối tượng thụ hưởng trực tiếp các kết quả, sản phẩm của các đề tài, dự án: doanh nghiệp, người dân,...

c) Nhóm giải pháp về chuyển giao, thương mại hóa, kết nối cung cầu:

- Đánh giá và lựa chọn các sản phẩm KH\&CN có khả năng chuyển giao, thương mại hóa, kết nối cung - cầu.

- Xây dựng chiến lược chuyển giao, thương mại hóa, kết nối cung - cầu cho các sản phẩm, nhóm sản phẩm tiềm năng.

- Huy động doanh nghiệp tham gia phối hợp triển khai nhằm chuyển giao, thương mại hóa và nhân rộng các kết quả, sản phẩm của đề tài, dự án. Trên cơ sở đó, chuyển giao kết quả nghiên cứu và sản phẩm của các đề tài, dự án cho các doanh nghiệp để tăng khả năng thương mại hóa sản phẩm, giúp thúc đẩy sự phát triển kinh tế và xã hội cho các địa phương.

- Có thể áp dụng cách thức chuyển giao theo từng giai đoạn của đề tài: Có sản phẩm đến phần nào thì chuyển giao cho địa phương và doanh nghiệp đến phần đó nhằm tiết kiệm thời gian cũng như điều chỉnh kết quả, sản phẩm cho phù hợp với điều kiện và nhu cầu thực tế.

d) Nhóm giải pháp về truyền thông:

Tăng cường quảng bá thông tin về kết quả, hiệu quả, đóng góp, tác động của các đề tài, dự án bằng cách xây dựng cổng thông tin để lãnh đạo, người dân, các nhà khoa học, doanh nghiệp và những ai quan tâm có thể truy cập. Lãnh đạo tỉnh sau khi truy cập, nếu thấy các kết quả phù hợp với tình hình phát triển kinh tế, xã hội và nhu cầu của tỉnh có thể đề xuất chuyển giao kết quả 
của đề tài, dự án, kể cả khi dự án không phải do tỉnh đặt hàng.

\section{Kết luận}

Tính hiệu quả của các đề tài, dự án được đánh giá dựa trên 20 chỉ thị thuộc 03 hợp phần: i) Hiệu quả về KH\&CN gồm 12 chỉ thị; ii) Hiệu quả về nguồn lực thực hiện gồm 04 chỉ thị; và iii) Hiệu quả về đào tạo gồm 04 chỉ thị. Kết quả đánh giá định lượng 08 đề tài, dự án KH\&CN thuộc Chương trình Tây Bắc cho thấy tính hiệu quả dao động trong khoảng $0,55-0,75$. Có 2 đề tài $(06 \mathrm{X}$ và $02 \mathrm{C})$ có tính hiệu quả cao và 6 đề tài, dự án được đánh giá là hiệu quả. Để nâng cao hiệu quả của các đề tài, dự án, cần áp dụng các nhóm giải pháp về $\mathrm{KH} \& \mathrm{CN}$; sự liên kết, phối hợp giữa các bên liên quan; chuyển giao, thương mại hóa, kết nối cung cầu; và truyền thông cho các kết quả, sản phẩm. Cần nghiên cứu, đề xuất thêm trọng số của một số chỉ thị đánh giá cho phù hợp với đề tài, dự án thuộc các lĩnh vực khác nhau cũng như đánh giá của các bên liên quan, đặc biệt là đối tượng sử dụng, ứng dụng trực tiếp kết quả, sản phẩm để tăng tính thiết thực, phù hợp của bộ chỉ thị đánh giá.

\section{Lời cảm ơn}

Nghiên cứu này được tài trợ bởi đề tài mã số KHCN-TB.27X/13-18 thuộc Chương trình KH \&CN phục vụ phát triển bền vững vùng Tây Bắc.

\section{Tài liệu tham khảo}

[1] I. Bartuševičienè and E. Šakalytè, Organizational assessment: Effectiveness vs. Efficiency, Social Transformations in Contemporary Society 1 (2013) 45-53.

[2] W. Zheng, B. Yang, G. McLean, Linking organizational culture, structure, strategy, and organizational effectiveness: Mediating role of knowledge management, Journal of Business Research 63(7) (2010) 763-771. https://doi.org/10. 1016/j.jbusres.2009.06.005.

[3] C. Donovan and S. Hanney, The "Payback Framework" explained. Research Evaluation 20 (2011) 181-183. https:// doi.org/10.3152/095820211X13118583635756.
[4] R. Banzi, L. Moja, V. Pistotti, A. Facchini and A. Liberati, Conceptual frameworks and empirical approaches used to assess the impact of health research: An overview of reviews, Health Research Policy and Systems 9 (2011) 26-36. https://dx.doi. org/10.1186\%2F1478-4505-9-26.

[5] H.P. McKenna, J. Daly, P. Davidson, C. Duffield, D. Jackson, RAE, ERA, Spot the difference, Int J Nurs Stud 49 (2012) 375-377. https://doi.org/10.1 016/j.ijnurstu.2011.11.013.

[6] C. Manville, S. Guthrie, M. Henham, B. Garrod, S. Sousa, A. Kirtley, S. Clarke and T. Ling, Assessing impact submissions for REF 2014: An evaluation. RAND, Prepared for HEFCE, SFC, HEFCW and DEL (2014). https://www.rand.org/pubs/research_ reports/RR1032.html (accessed 30 December 2019).

[7] REF2014, 2010. Decisions on Assessing Research Impact. https://www.ref.ac.uk/2014/media/ref/ content/pub/decisionsonassessingresearchimpact/0 1_11.pdf (accessed 30 December 2019).

[8] CAHS (Canadian Academy of Health Sciences Panel on Return on Investment in Health Research), Making an Impact: A Preferred Framework and Indicators to Measure Returns on Investment in Health Research (Publish online), 2009. http://www. cahsacss.ca/wp_content/uploads/2011/ 09/ROI_FullReport .pdf (accessed 30 December 2019).

[9] M. Duryea, M. Hochman and A. Parfitt, Measuring the Impact of Research, Research Global, 27, 8-9 (Publish online) (2007). http://facdent.hku.hk/docs/ ResGlob2007.pdf (accessed 30 December 2019).

[10] S. Morton, Progressing research impact assessment: A 'contributions' approach. Research Evaluation 24 (2015) 405-419. https://doi.org/10.1093/reseval/rvv016.

[11] S.H. Oh, H.Y. Lim, B. Kim, Strategy to Promote the Effectiveness of Technology Transfer of National R\&D Programs in Korea: Seen through the G7 Leading Technology Development Program, Procedia Computer Science 91 (2016) 221 - 229. https://doi.org/10.1016/j.procs.2016.07.061.

[12] H. Lee, Y. Park, H. Choi, Comparative evaluation of performance of national $R \& D$ programs with heterogeneous objectives: A DEA approach, European Journal of Operational Research 196 (2009) 847-855. https://doi.org/10.1016/j.ejor.2008. 06.016 .

[13] P. Patunakul, Y. H. Kwak, O. Zwikael, M. Liu, What impacts the performance of large-scale government projects?, International Journal of Project Management 34 (2016) 452-466. https:// doi.org/10.1016/j.ijproman.2015.12.001. 
[14] B. Bozeman, J. Youtie, Socio-Economic impacts and public value of government-funded research: Lessons from four US National Science Foundation initiatives. Research Policy 46(8) (2017) 1387-1398. https://doi.org/10.1016/j.respol. 2017.06.003.

[15] B.P. Cozzarin, Data and the measurement of R\&D program impacts, Evaluation and Program Planning 31 (2008) 284-298. https://doi.org/10. 1016/j.evalprogplan. 2008.03.004.

[16] J. Han, M. Kamber, J. Pei, Data mining - Concepts and Techniques, 3rd edition, Elsevier Inc, USA (2012).
[17] UNDP, Human development report, United Nations Development Program (2006).

[18] M. Buxton and S. Hanney, How can payback from health services research be assessed? J Health Serv Res Policy 1(1) (1996) 35-43. https://doi.org/10. $1177 \% 2 F 135581969600100107$.

[19] S. Wooding, S. Hanney, M Buxton, J. Grant, The returns from arthritis research Volume 1: approach, analysis and recommendations, A report prepared for the Arthritis Research Campaign, RAND Europe (2004) 